\title{
Controlled Attenuation Parameter for Assessment of Hepatic Steatosis in Indian Patients
}

\author{
Gyanranjan Rout ", Saurabh Kedia*, Baibaswata Nayak*, Rajni Yadav ${ }^{\dagger}$, Prasenjit Das ${ }^{\dagger}$, Subrat K. Acharya*, \\ Deepak Gunjan ", Vishwajeet Singh ${ }^{\ddagger}$, Mousumi Mahanta ${ }^{*}$, Swatantra Gupta ${ }^{*}$, Sandeep Aggarwal ${ }^{\S}$, Shalimar \\ *Department of Gastroenterology, All India Institute of Medical Sciences, New Delhi, India, 'Department of Pathology, All India Institute of \\ Medical Sciences, New Delhi, India, ${ }^{\ddagger}$ Department of Biostatistics, All India Institute of Medical Sciences, New Delhi, India and ${ }^{\S}$ Department of \\ Surgery, All India Institute of Medical Sciences, New Delhi, India
}

\begin{abstract}
Background/Aims: The gold standard method for measurement of hepatic steatosis is liver histology. Controlled Attenuation Parameter (CAP) can measure hepatic steatosis non-invasively. We aimed to assess the accuracy of CAP for detection of hepatic steatosis. Methods: A total of 462 patients (May 2012-January 2017)-89 nonalcoholic fatty liver disease, 182 chronic hepatitis $B, 88$ chronic hepatitis $C$ and 103 patients with other etiologies who underwent simultaneous liver biopsy and CAP estimation using Transient Elastography (TE) were included. Steatosis was graded as S0: steatosis in 0-5\% of hepatocytes, S1: 6-33\%, S2: 34-66\% and S3: 67$100 \%$. Receiver Operating Characteristic (ROC) curves were plotted to evaluate the accuracy of CAP in detecting hepatic steatosis. Predictors of CAP were assessed by multivariate linear regression model. Results: The mean age \pm SD was $33.8 \pm 11.6$ years; $296(64.1 \%)$ were males. On liver histology, steatosis grades S0, S1, S2 and S3 were seen in $331(71.6 \%), 74(16.0 \%), 39(8.4 \%)$ and $18(3.9 \%)$, respectively. The median CAP (IQR) values for S0, S1, S2, and S3 steatosis were $206(176-252) \mathrm{dB} / \mathrm{m}, 295$ (257-331) dB/m, 320 (296-356) dB/m, and 349 (306-363) $\mathrm{dB} / \mathrm{m}$, respectively. For estimation of $\geq \mathrm{S} 1, \geq \mathrm{S} 2$, and $\geq \mathrm{S3}$ using CAP, AUROC were 0.879, 0.893, and 0.883, respectively. In multivariate analysis, only $\mathrm{BMI}(\mathrm{OR} 1.18 ; \mathrm{CI}, 1.11-1.26, P<0.001)$ and grade of hepatic steatosis (grade 1, OR, 3.94; 95\% CI, 1.58-9.84, $P=0.003$; grade 2, OR 42.04; 95\% CI, 4.97-355.31, $P=0.001$ and grade 3, OR 35.83; 95\% CI 4.31-297.61, $P=0.001$ ) independently predicted CAP. Conclusions: CAP detects hepatic steatosis with good accuracy in Indian patients with various etiologies. (J Clin EXP HePATOL 2019;9:13-21)
\end{abstract}

$\mathrm{H}$ epatic steatosis is commonly seen in Non-Alcoholic Fatty Liver Disease (NAFLD), Alcoholic Liver Disease (ALD), and Chronic Hepatitis C (CHC) patients. ${ }^{1,2}$ The presence of hepatic steatosis is associated with treatment failure in Chronic Hepatitis B (CHB) patients, ${ }^{3}$ progression of hepatic fibrosis ${ }^{4}$ and development of hepatocellular carcinoma in $\mathrm{CHC}^{5}$ In addition, hepatic steatosis is also associated with metabolic syndrome and its complications. ${ }^{6}$ Therefore, estimation of hepatic steatosis is important in the management of patients. Till now, the gold standard for assessment of

Keywords: liver biopsy, NAFLD, fibrosis, hepatitis B virus, hepatitis C virus

Received: 8 December 2017; Accepted: 20 February 2018; Available online: 14 March 2018

Address for correspondence: Shalimar, Department of Gastroenterology, All India Institute of Medical Sciences, Room No 127, 1st Floor, Human Nutrition Unit, New Delhi 110029, India. Tel.: +91 1126546643.

E-mail: drshalimar@yahoo.com

Abbreviations: ALT: Alanine Aminotransferase; AST: Aspartate Aminotransferase; AUROC: Area Under Receiver Operating Characteristics Curves; BMI: Body Mass Index; CAP: Controlled Attenuation Parameter; CHB: Chronic Hepatitis B; CHC: Chronic Hepatitis C; IQR: Interquartile Range; LSM: Liver Stiffness Measurement; NAFLD: Non-Alcoholic Fatty Liver Disease; SD: Standard Deviation https://doi.org/10.1016/j.jceh.2018.02.010 hepatic steatosis is liver biopsy. Liver biopsy is an invasive procedure with complications like pain, bleeding, sampling variability, and even a small risk of death $(0.01 \%)$. Moreover, it is not feasible to perform repeated biopsies to assess for changes in steatosis on follow-up.

Ultrasonography (US), Computed Tomography (CT), Magnetic Resonance Imaging (MRI) and Magnetic Resonance Spectroscopy (MRS) are some of the imaging methods available for noninvasive assessment of hepatic steatosis. ${ }^{8}$ However, these techniques have limitations, including high operator-dependency of US, radiation exposure during $\mathrm{CT}$, high cost and limited availability of MRI and MRS. ${ }^{9-11}$ Thus, there remains an unmet need for a low cost, easily available, accurate and non-invasive method for detection of hepatic steatosis. Recent data suggests that Controlled Attenuation Parameter (CAP) correlates with steatosis on liver biopsy. ${ }^{12}$ It can be used for steatosis detection and quantification. Prior studies have evaluated its accuracy in NAFLD, ${ }^{13}$ ALD, HCV infection $^{14}$ and patients with CHB infection. ${ }^{15}$ There is a paucity of Indian data on the predictive value of CAP for hepatic steatosis on liver biopsy. Therefore, the objectives of this study were: (i) to assess the diagnostic accuracy of CAP in assessing hepatic steatosis; and (ii) to assess the various factors affecting CAP values. 


\section{PATIENTS AND METHODS}

\section{Inclusion and Exclusion Criteria}

We searched our prospectively maintained database for all patients undergoing liver biopsy and Fibroscan after May 2012. The FibroScan touch 502 (Echosens, Paris, France) was available at our center from November 2013. All consecutive patients, who underwent concomitant liver biopsy and CAP estimation in the department of Gastroenterology, All India Institute of Medical Sciences, New Delhi, India between May 2012 to January 2017, were included in this study.

The etiology of underlying liver disease included CHB, CHC, NAFLD, and a miscellaneous group (which included autoimmune hepatitis, non-cirrhotic portal fibrosis (NCPF) and cryptogenic etiology). An adequate liver biopsy sample was defined as one with atleast $15 \mathrm{~mm}$ of sample tissue and containing atleast six portal tracts or more. Patients were excluded from the study if they had co-infection with other hepatotropic viruses or HIV, bilirubin $\geq 5 \mathrm{mg} / \mathrm{dl}$ or Aspartate Aminotransferase (AST)/ Alanine Aminotransferase (ALT) levels 10 times the upper limit of normal in the last 6 months, ascites, previous liver surgery or liver transplantation or inadequate liver biopsy sample.

\section{Data Collection}

The clinical records of all patients were retrospectively retrieved from a prospectively maintained database. The following details were noted in each case: age, sex, alcohol intake, the presence of hypertension and diabetes, Body Mass Index (BMI), various laboratory test results including hemoglobin, platelet count, AST, ALT, fasting blood sugar, fasting lipid profile \{total cholesterol, Low-Density Lipoprotein (LDL), Very Low-Density Lipoprotein (VLDL), Triglycerides (TG) and High-Density Lipoprotein (HDL)\} levels. Tests for hepatitis B surface antigen, antibodies to hepatitis $\mathrm{C}$ virus (anti-HCV), and Human Immunodeficiency Virus (HIV) were done using standard commercially available enzyme immunoassays.

\section{Liver Stiffness Measurement and Determination of CAP}

Liver Stiffness Measurement (LSM) and CAP measurements were performed on a FibroScan ${ }^{\circledR}$ touch 502 (Echosens, Paris, France) by trained personnel who were blinded to the clinical data of patients. All LSM and CAP measurements were done within 4 weeks prior to the liver biopsy. The FibroScan ${ }^{\circledR}$ was performed in a fasting state on the right lobe of the liver through the intercostal space. All measurements were done using $\mathrm{M} / \mathrm{XL}$ probe as per manufacturer's recommendations. As a routine, we used XL probe for measurements in obese patients with BMI above $30 \mathrm{~kg} / \mathrm{m}^{2}$. Ten successful acquisitions were performed on each patient. The interquartile range (IQR) was defined as the index of the intrinsic variability of LSM and CAP values corresponding to the interval containing $50 \%$ of the valid measurements between the 25 th and 75 th percentiles. As an indicator of variability, the ratios of the IQR of LSM and CAP values to the median values (IQR/M) were calculated. The measurements were considered reliable if 10 valid acquisitions with IQR/M of LSM less than 0.3 were obtained. Failure of transient elastography was defined as the inability to obtain valid LSM or CAP value.

\section{Liver Biopsy Examination}

Percutaneous liver biopsy was performed using a $16 \mathrm{G}$ Menghini's liver biopsy needle under local anesthesia and ultrasound guidance. The liver biopsy was done to document the grade and stage of liver disease in hepatitis $\mathrm{B}$, hepatitis $\mathrm{C}$ and NAFLD. In other etiologies, biopsies were done to confirm the diagnosis. Liver biopsy was assessed by 2 experienced pathologists who were blinded to the clinical and CAP data. A liver biopsy sample was considered adequate if it was longer than $15 \mathrm{~mm}$ and contained six portal tracts or more. Liver fibrosis was evaluated semi-quantitatively according to the METAVIR scoring system as follows: F0, no fibrosis; F1, portal fibrosis without septa; F2, portal fibrosis and few septa; F3, numerous septa without cirrhosis; and F4, cirrhosis. ${ }^{16}$ Steatosis was categorized by visual assessment as S0, steatosis in $0-5 \%$; S1, steatosis in $6-33 \%$; $\mathrm{S} 2$, in $34-66 \%$; and S3, $67-100 \%$ of hepatocytes. ${ }^{2}$

\section{Statistical Analysis}

Continuous variables were expressed as mean $\pm \mathrm{SD}$ or median (interquartile range), as appropriate. Continuous variables were compared by Student's $t$-test or MannWhitney test, as appropriate, or ANOVA test (for more than two group comparisons). Categorical variables were compared by chi-square test or Fisher's exact test. Linear regression (simple-i.e. univariate, and multiple-i.e. multivariate) was used to assess the association between CAP and other relevant variables. The accuracy of CAP in predicting histological steatosis grade was evaluated by plotting area under the receiver operating characteristic curves (AUROC). The threshold cut-offs of CAP values were identified by the ROC curve analysis and, based on these cut-offs, the different diagnostic measures such as sensitivity, specificity, positive predictive value, negative predictive value, positive likelihood ratio, and negative likelihood ratio were reported. AUROCs were interpreted as follows: $0.90-1.0=$ excellent; $0.80-0.90=$ good; $0.70-$ 0.80 = fair; $<0.70=$ poor. All statistical tests were twosided. Statistical significance was taken as $P<0.05$. Statistical analysis was performed using SPSS (SPSS version 20.0; SPSS Inc., Chicago, IL, USA). 


\section{RESULTS}

\section{Baseline Characteristics}

Out of a total of 504 patients who underwent Fibroscan ${ }^{\circledR}$ (LSM and CAP) and biopsy during the study period, 42 were excluded-inadequate biopsy in 16, invalid fibroscan ${ }^{\circledR}$ in 13 , HIV co-infection in 3, AST/ALT levels 10 times the upper limit of normal in 8 , and previous liver surgery in 2 patients.

Finally, a total of 462 patients were included in the analysis. The mean age of these patients was $33.8 \pm 11.8$ years and $296(64.1 \%)$ were males. The etiologies were NAFLD in 89 (19.3\%), HBV in 182 (39.4\%), HCV in 88 (19.0\%) and miscellaneous in 103 (22.3\%). The miscellaneous group comprised of autoimmune hepatitis in 46 patients, NCPF in 13, and cryptogenic in 44 . The median BMI was $23.4 \mathrm{~kg} / \mathrm{m}^{2}$ (IQR, 20.8-26.4). The median (IQR) CAP value was $229.5(189.0-290.0) \mathrm{dB} / \mathrm{m}$, and median LSM was 6.8 (5.3-9.1) $\mathrm{kPa}$. The baseline characteristics, laboratory parameters, liver biopsy (hepatic steatosis and hepatic fibrosis) details, and fibroscan ${ }^{\circledR}$ (LSM and CAP) values are shown in Table 1. Patients with NAFLD were more likely to be older, and as expected had higher BMI, as well as co-morbidities such as hypertension, metabolic syndrome, diabetes mellitus or dyslipidemia (elevated values of total serum cholesterol and serum TGs). On liver histology, higher grades of steatosis and fibrosis were seen in patients with NAFLD as compared to other etiologies (Table 1). The CAP values were higher in patients with NAFLD as compared to other etiologies of CLD (Table 1). The median (IQR) CAP values in patients with NAFLD, $\mathrm{CHB}, \mathrm{CHC}$ and miscellaneous etiologies were 325.0 (300.0-361.5), 212.5 (184.7-252.0), 202.0 (171.5-249.7) and 231.0 (190.0-274.0), respectively $(P<0.001)$.

\section{Univariate and Multivariate Analysis of Factors Predicting CAP}

Older age, female sex, underlying etiology of liver disease, high BMI, metabolic syndrome, low bilirubin, low AST, low ALT, low serum alkaline phosphatase, high total cholesterol, high TG, high LDL, high HDL, high VLDL and higher grade of hepatic steatosis (on liver biopsy) were associated with CAP in univariate analysis. However, only BMI (OR 1.18; CI, 1.11-1.26, $P<0.001$ ) and hepatic steatosis grade (grade S1, OR, 3.94; 95\% CI, 1.58-9.84, $P=0.003$; grade S2, OR 42.04; 95\% CI, 4.97-355.31, $P=0.001$ and grade S3, OR 35.83; 95\% CI 4.31-297.61, $P=0.001)$ independently predicted CAP on multivariate analysis (Table 2).

\section{CAP Values for Hepatic Steatosis Assessment}

The overall CAP values for each grade of hepatic steatosis are shown in Figure 1. Among NAFLD patients, the median (IQR) CAP values in patients with histological steatosis grades S1, S2, and S3 were 314.0 (281.0354.0), 334.5 (313.0-359.5) and 360.5 (316.7-363.7), respectively $(P=0.031)$. Among $\mathrm{CHB}$ patients, the median (IQR) CAP values in patients with histological steatosis S0, S1, S2, and S3 were 206 (180.0-243.0), 234 (213.7-287.7), 305 (289.7-337.2) and 332 (265.5-368.5), respectively $(P<0.001)$. Among CHC patients, the median (IQR) CAP values in patients with histological steatosis S0, S1, S2, and S3 were 199 (166.7-228.2), 267 (221.0279.5), 275.5 (208.7-288.2) and 288 (288-288), respectively $(P=0.006)$. Among miscellaneous etiologies of CLD, the median (IQR) CAP values in patients with histological steatosis S0, S1, and S2 were 228.5 (189.7-273.2), $254(136.0-277.5)$ and 303 (303-303), respectively $(P=0.503)$. There was no patient with $\mathrm{S} 3$ steatosis in the miscellaneous group.

For the overall cohort, AUROC of CAP for prediction of $\geq \mathrm{S} 1, \geq \mathrm{S} 2$, and $\mathrm{S} 3$ grades of hepatic steatosis were 0.879 , 0.893 , and 0.883 , respectively (Figure 2). Overall, the CAP cut-off values for estimation of hepatic steatosis grades $\geq \mathrm{S} 1, \geq \mathrm{S} 2$, and S3 were $263 \mathrm{~dB} / \mathrm{m}, 287 \mathrm{~dB} / \mathrm{m}$, and $296 \mathrm{~dB} /$ $\mathrm{m}$, respectively. The CAP cut-off values for differentiating various grades of hepatic steatosis were different among various etiologies. Among NAFLD patients, CAP cut-off values for estimation of hepatic steatosis grades $\geq \mathrm{S} 2$, and $\mathrm{S} 3$ were $324 \mathrm{~dB} / \mathrm{m}$ and $348 \mathrm{~dB} / \mathrm{m}$, respectively. Among CHB patients, CAP cut-off values for estimation of hepatic steatosis grades $\geq \mathrm{S} 1, \geq \mathrm{S} 2$, and $\mathrm{S} 3$ were $232 \mathrm{~dB} / \mathrm{m}$, $288 \mathrm{~dB} / \mathrm{m}$, and $304 \mathrm{~dB} / \mathrm{m}$, respectively. Among $\mathrm{CHC}$ patients, CAP cut-off values for estimation of hepatic steatosis grades $\geq \mathrm{S} 1, \geq \mathrm{S} 2$, and $\mathrm{S} 3$ were $252 \mathrm{~dB} / \mathrm{m}$, $272 \mathrm{~dB} / \mathrm{m}$, and $286 \mathrm{~dB} / \mathrm{m}$, respectively.

\section{CAP for Hepatic Steatosis Assessment- Influence of Stage of Fibrosis}

There was no significant difference in CAP values in different stages of fibrosis with similar grade of steatosis. Among patients with S0 steatosis, median (IQR) CAP values in patients with histological fibrosis stages $\mathrm{F} 0$, F1, F2, F3 and F4 were $200.0(171.2-252.0) \mathrm{dB} / \mathrm{m}, 210.0$ (178.5-252.0) dB/m, $220.0(194.5-260.5) \mathrm{dB} / \mathrm{m}, 202$ (167.0-253.0) dB/m, and $206.5(180.2-234.0) \mathrm{dB} / \mathrm{m}$, respectively $(P=0.393)$. In $\mathrm{S} 1$ steatosis, median (IQR) CAP values in patients with histological fibrosis stages F0, F1, F2, F3 and F4 were $271.0(234.0-315.0) \mathrm{dB} / \mathrm{m}$, 300.5 (254.0-347.5), 343.5 .0 (307.5-370.7) dB/m, 297.0 (274.2-348.7) dB/m, and 300.0 (267.0-333.0) dB/m, respectively $(P=0.151)$. In $\mathrm{S} 2$ steatosis, median (IQR) $\mathrm{CAP}$ values in patients with histological fibrosis stages F0, F1, F2, F3 and F4 were 331.0 (317.0-358.0) dB/m, 338.0 (303.0-363.0) dB/m, 320.0 (292.0-367.0) dB/m, $318.0(276.2-345.5) \mathrm{dB} / \mathrm{m}$, and $298.0(278.0-322.7) \mathrm{dB} /$ $\mathrm{m}$, respectively $(P=0.524)$. In $\mathrm{S} 3$ steatosis, median (IQR) CAP values in patients with histological fibrosis stages F0, 
Table 1 Baseline Demographic, and Laboratory Parameters of Patients With Various Etiologies of Chronic Liver Disease.

\begin{tabular}{|c|c|c|c|c|c|c|}
\hline Parameter & $\begin{array}{c}\text { Overall } \\
(n=462)\end{array}$ & $\begin{array}{c}\text { NAFLD } \\
(n=89)\end{array}$ & $\begin{array}{c}\text { CHB } \\
(n=182)\end{array}$ & $\begin{array}{c}\text { CHC } \\
(n=88)\end{array}$ & $\begin{array}{l}\text { Miscellaneous } \\
\quad(n=103)\end{array}$ & $P$-value \\
\hline Age, years (mean $\pm S D$ ) & $33.8( \pm 11.6)$ & $38.6( \pm 9.9)$ & $30.2( \pm 11.2)$ & $34.7( \pm 11.6)$ & $35.2( \pm 11.9)$ & $<0.001^{*, \S, \text { ฯ }}$ \\
\hline Male:female & $\begin{array}{l}296(64.1 \%): 166 \\
(35.9 \%)\end{array}$ & $\begin{array}{l}43(48.3 \%): 46 \\
(51.7 \%)\end{array}$ & $\begin{array}{l}147 \text { (80.8\%):35 } \\
(19.2 \%)\end{array}$ & $\begin{array}{l}62(70.5 \%): 26 \\
(29.5 \%)\end{array}$ & $\begin{array}{l}44(42.7 \%): 59 \\
(57.3 \%)\end{array}$ & $<0.001^{*, \dagger, \uparrow, \|}$ \\
\hline BMI $\left(\mathrm{kg} / \mathrm{m}^{2}\right)$ & $\begin{array}{l}23.4 \\
(20.8-26.4)\end{array}$ & $\begin{array}{l}29.2 \\
(25.4-40.3)\end{array}$ & $\begin{array}{l}21.9 \\
(19.6-24.9)\end{array}$ & $\begin{array}{l}22.7 \\
(20.5-24.8)\end{array}$ & $\begin{array}{l}24.2 \\
(21.4-28.4)\end{array}$ & $<0.001^{*, \uparrow, \ddagger, \boldsymbol{\uparrow}, \|}$ \\
\hline Hypertension $(n=381)$ & $25(6.6 \%)$ & $17(36.2 \%)$ & $2(1.1 \%)$ & $2(2.3 \%)$ & $4(5.6 \%)$ & $<0.001^{*, \dagger, \ddagger}$ \\
\hline Diabetes $(n=392)$ & $59(15.1 \%)$ & $31(55.4 \%)$ & $8(4.5 \%)$ & 7 (8.2\%) & $13(18.1 \%)$ & $<0.001^{*, \dagger, \downarrow, q}$ \\
\hline $\begin{array}{l}\text { Metabolic syndrome } \\
(n=432)\end{array}$ & $62(14.3 \%)$ & $43(50 \%)$ & $0(0 \%)$ & $0(0 \%)$ & $19(20.2 \%)$ & $<0.001^{*, \dagger, \ddagger, \uparrow, \|}$ \\
\hline Bilirubin, mg/dl & $0.6(0.5-0.8)$ & $0.6(0.5-0.8)$ & $0.6(0.5-0.8)$ & $0.6(0.5-0.8)$ & $0.7(0.5-1.1)$ & $0.002^{\ddagger, \uparrow, \|}$ \\
\hline AST, IU/L & $40(28-62)$ & $31.0(23-57)$ & $34(28-51)$ & $53(42-88)$ & $40.0(23-71)$ & $<0.001^{\dagger, \S, \|}$ \\
\hline$A L T, I U / L$ & $43(26-84)$ & $31(22-69)$ & $43(29-69)$ & $76(52-133)$ & $36(21-72)$ & $<0.001^{\dagger, \S, \|}$ \\
\hline SAP, IU/L & $191(122-262)$ & $125(83-215)$ & $202(145-257)$ & 204 (143-269) & $197(109-351)$ & $<0.001^{*, \ddagger, \uparrow, \|}$ \\
\hline Albumin, g/dl (mean $\pm \mathrm{SD}$ ) & $4.4( \pm 0.6)$ & $4.2( \pm 0.6)$ & $4.6( \pm 0.6)$ & $4.6( \pm 0.5)$ & $4.0( \pm 0.69)$ & $<0.001^{*, \dagger, \uparrow, \|}$ \\
\hline $\begin{array}{l}\text { Hemoglobin, g/dl } \\
(\text { mean } \pm \text { SD) }\end{array}$ & $13.1( \pm 2.0)$ & $12.9( \pm 1.9)$ & $13.7( \pm 1.8)$ & $13.6( \pm 1.7)$ & $13.6( \pm 1.7)$ & $<0.001^{*, \ddagger \text {, }}$ \\
\hline $\mathrm{TLC}, / \mathrm{mm}^{3}($ mean $\pm \mathrm{SD})$ & $7214( \pm 2231)$ & $8281( \pm 2470)$ & $7034( \pm 1828)$ & $7324( \pm 2102)$ & $7324( \pm 2121)$ & $<0.001^{*, t, \ddagger}$ \\
\hline $\begin{array}{l}\text { Platelets } \times 10^{3}, / \mathrm{mm}^{3} \\
(\text { mean } \pm \mathrm{SD})\end{array}$ & $195( \pm 85)$ & $231( \pm 92)$ & $182( \pm 67)$ & $182( \pm 60)$ & $182( \pm 60)$ & $<0.001^{*, \dagger}$ \\
\hline $\begin{array}{l}\text { Total cholesterol, mg/dl } \\
\text { (mean } \pm \mathrm{SD})\end{array}$ & $166.5( \pm 38.5)$ & $177.4( \pm 38.1)$ & $158.9( \pm 37.1)$ & $161.4( \pm 40.1)$ & $161.4( \pm 40.1)$ & $0.009^{*}$ \\
\hline $\begin{array}{l}\text { Triglycerides, } \mathrm{mg} / \mathrm{dl} \\
(\text { mean } \pm \mathrm{SD})\end{array}$ & $126.5( \pm 54.7)$ & $146.6( \pm 51.8)$ & $112.0( \pm 47.8)$ & $95.6( \pm 33.1)$ & $117.4( \pm 57.9)$ & $<0.001^{*, \dagger}$ \\
\hline $\mathrm{LDL}, \mathrm{mg} / \mathrm{dl}($ mean $\pm \mathrm{SD})$ & $99.0( \pm 31.8)$ & $104.4( \pm 32.4)$ & $95.8( \pm 29.7)$ & $95.6( \pm 33.1)$ & $95.6( \pm 33.1)$ & 0.287 \\
\hline $\mathrm{HDL}, \mathrm{mg} / \mathrm{dl}($ mean $\pm \mathrm{SD})$ & $42.1( \pm 8.6)$ & $43.9( \pm 9.4)$ & $40.3( \pm 7.8)$ & $42.0( \pm 4.9)$ & $42.0( \pm 4.9)$ & $0.032^{*}$ \\
\hline VLDL, mg/dl (mean $\pm \mathrm{SD})$ & $23.8( \pm 10.3)$ & $28.0( \pm 9.7)$ & $20.9( \pm 9.9)$ & $23.5( \pm 13.2)$ & $23.5( \pm 13.2)$ & $<0.001^{*, \ddagger}$ \\
\hline Hepatic fibrosis (METAVIR) & & & & & & $<0.001^{*, \S, \uparrow, \|}$ \\
\hline 0 & $177(38.3 \%)$ & $22(24.7 \%)$ & $92(50.5 \%)$ & 30 (34.1\%) & $33(32 \%)$ & \\
\hline 1 & $114(24.7 \%)$ & $26(29.2 \%)$ & $48(26.4 \%)$ & $24(27.3 \%)$ & $16(15.5 \%)$ & \\
\hline 2 & $59(12.8 \%)$ & $15(16.9 \%)$ & $23(12.6 \%)$ & $12(13.6 \%)$ & $9(8.7 \%)$ & \\
\hline 3 & $51(11.0 \%)$ & $11(12.4 \%)$ & 9 (4.9\%) & $14(15.9 \%)$ & 17 (16.5\%) & \\
\hline 4 & $61(13.2 \%)$ & $15(16.9 \%)$ & $10(5.5 \%)$ & $8(9.1 \%)$ & $28(27.2 \%)$ & \\
\hline Grade of hepatic steatosis & & & & & & $<0.001^{*, \dagger, \ddagger}$ \\
\hline 0 & $331(71.6 \%)$ & - & $155(85.2 \%)$ & $78(88.6 \%)$ & $98(95.1 \%)$ & \\
\hline 1 & $74(16.0 \%)$ & 49 (55.1\%) & $16(8.8 \%)$ & $5(5.7 \%)$ & $4(3.9 \%)$ & \\
\hline 2 & $39(8.4 \%)$ & $28(31.5 \%)$ & $6(3.3 \%)$ & $4(4.5 \%)$ & $1(1.0 \%)$ & \\
\hline 3 & $18(3.9 \%)$ & $12(13.5 \%)$ & $5(2.7 \%)$ & $1(1.1 \%)$ & $0(0 \%)$ & \\
\hline LSM, kPa & $6.8(5.3-9.1)$ & $8.2(6.0-11.9)$ & $6.1(4.9-7.8)$ & $6.3(5.0-8.6)$ & $8.8(6.0-15.1)$ & $<0.001^{*, \uparrow, \|}$ \\
\hline $\mathrm{CAP}, \mathrm{dB} / \mathrm{m}$ & $\begin{array}{l}229.5 \\
(189.0-290.0)\end{array}$ & $\begin{array}{l}325.0 \\
(300.0-361.5)\end{array}$ & $\begin{array}{l}212.5 \\
(184.7-252.0)\end{array}$ & $\begin{array}{l}202.0 \\
(171.5-249.7)\end{array}$ & $\begin{array}{l}231.0 \\
(190.0-274.0)\end{array}$ & $<0.001^{*, \dagger, \ddagger, \|}$ \\
\hline
\end{tabular}

Note: All values are expressed as median (IQR) or $n(\%)$ unless otherwise specified.

*Significant between NAFLD and CHB.

'Significant between NAFLD and CHC.

¥Significant between NAFLD and miscellaneous.

¿Significant between $\mathrm{CHB}$ and $\mathrm{CHC}$.

ISignificant between CHB and miscellaneous.

"Significant between $\mathrm{CHC}$ and miscellaneous.

Abbreviations: BMI, body mass index; AST, aspartate aminotransferase; ALT, alanine aminotransferase; SAP, serum alkaline phosphatase; TLC, total leukocyte count; LDL, low-density lipoprotein; HDL, high-density lipoprotein; VLDL, very low-density lipoprotein; LSM, liver stiffness measurement; IQR, interquartile range; CAP, controlled attenuation parameter. 
Table 2 Univariate and Multivariate Analysis of Factors Predicting Controlled Attenuation Parameter.

\begin{tabular}{|c|c|c|c|c|}
\hline Variables & Unadjusted OR (95\% CI) & $P$-value & Adjusted OR (95\% Cl) & $P$-value \\
\hline Age, years & 1.041 (1.024-1.059) & $<0.001$ & & \\
\hline Female sex & 1.905 (1.288-2.816) & 0.001 & & \\
\hline \multicolumn{5}{|l|}{ Diagnosis } \\
\hline Miscellaneous & 1 & & & \\
\hline NAFLD & 23.773 (9.918-56.985) & $<0.001$ & & \\
\hline $\mathrm{CHB}$ & $0.553(0.322-0.952)$ & 0.033 & & \\
\hline $\mathrm{CHC}$ & $0.486(0.249-0.949)$ & 0.035 & & \\
\hline $\mathrm{BMI}, \mathrm{kg} / \mathrm{m}^{2}$ & 1.239 (1.177-1.304) & $<0.001$ & $1.180(1.111-1.260)$ & $<0.001$ \\
\hline Metabolic syndrome present & 38.382 (13.587-108.429) & $<0.001$ & & \\
\hline Bilirubin, mg/dl & $0.505(0.322-0.793)$ & 0.003 & & \\
\hline AST, IU/I & 0.995 (0.990-0.999) & 0.024 & & \\
\hline ALT, IU/I & $0.996(0.992-0.999)$ & 0.018 & & \\
\hline SAP, IU/I & $0.994(0.993-0.997)$ & $<0.001$ & & \\
\hline Albumin, g/dl & $0.754(0.560-1.016)$ & 0.063 & & \\
\hline Total cholesterol, mg/dl & 1.012 (1.005-1.019) & $<0.001$ & & \\
\hline Triglycerides, mg/dl & 1.009 (1.000-1.014) & $<0.001$ & & \\
\hline LDL, mg/dl & $1.012(1.004-1.020)$ & 0.002 & & \\
\hline $\mathrm{HDL}, \mathrm{mg} / \mathrm{dl}$ & $1.046(1.015-1.077)$ & 0.004 & & \\
\hline VLDL, mg/dl & $1.056(1.027-1.087)$ & $<0.001$ & & \\
\hline LSM, mg/dl & 1.001 (0.982-1.019) & 0.951 & & \\
\hline Fibrosis, present & $1.051(0.921-1.200)$ & 0.730 & & \\
\hline \multicolumn{5}{|l|}{ Grade of hepatic steatosis } \\
\hline Grade 0 & 1 & & 1 & \\
\hline Grade 1 & $10.328(5.820-18.328)$ & $<0.001$ & $3.941(1.580-9.841)$ & 0.003 \\
\hline Grade 2 & 75.708 (17.786-322.260) & $<0.001$ & $42.042(4.971-355.312)$ & 0.001 \\
\hline Grade 3 & 69.569 (9.092-532.328) & $<0.001$ & $35.830(4.312-297.611)$ & 0.001 \\
\hline
\end{tabular}

Abbreviations: NAFLD, non-alcoholic fatty liver disease; CHB, chronic hepatitis B; CHC, chronic hepatitis C; BMI, body mass index; AST, aspartate aminotransferase; ALT, alanine aminotransferase; SAP, serum alkaline phosphatase; LDL, low-density lipoprotein; HDL, high-density lipoprotein; VLDL, very low-density lipoprotein; LSM, liver stiffness measurement.

F1, F2, F3 and F4 were $309.0(276.0-363.0) \mathrm{dB} / \mathrm{m}, 330.5$ (314.0-347.0) dB/m, 362.0 (324.0-383.0) dB/m, 349.0 (332.0-366.0) $\mathrm{dB} / \mathrm{m}$, and 343.5 (325.0-362.0) $\mathrm{dB} / \mathrm{m}$ respectively $(P=0.516)$ (Figure 3$)$.

The AUROCs of CAP value for various grades of hepatic steatosis $-\geq \mathrm{S} 1, \geq \mathrm{S} 2$, and $\geq \mathrm{S} 3$ for various grades of fibrosis are shown in Table 3 and Figure 2. Overall, CAP was a good test for differentiating $\geq \mathrm{S} 1, \geq \mathrm{S} 2$, and $\geq \mathrm{S} 3$, irrespective of the underlying fibrosis in the liver. The specificity, sensitivity, positive predictive value, negative predictive value, positive likelihood ratio, and negative likelihood ratio for optimal CAP cut-off values are shown in Table 3.

\section{CAP for Differentiating Various Grades of Hepatic Steatosis}

ROC curves were made to assess the ability of CAP to differentiate various individual grades of hepatic steatosis.
The details of AUROC, optimal cut-off values, sensitivity, specificity, PPV, NPV, positive and negative LR are shown in Table 4. CAP was excellent (AUROC > 0.90) in differentiating between S0/S2 (AUROC, 0.936) and S0/S3 grades (AUROC, 0.954) of hepatic steatosis. CAP was good (AUROC $>0.80$ ) in differentiating between $S 0 / S 1$ grades (AUROC, 0.831) and fair in differentiating between S1/S3 grades (AUROC, 0.715 ) of hepatic steatosis. CAP was poor in differentiating between S1/S2 grades (AUROC, 0.659) and S2/S3 grades (AUROC, 0.600) of hepatic steatosis.

\section{DISCUSSION}

The present study included a large number of patients with different etiologies of chronic liver disease who underwent simultaneous assessment of fibrosis and hepatic steatosis by liver histology and Fibroscan (LSM and CAP). The results indicate that CAP, a novel non- 


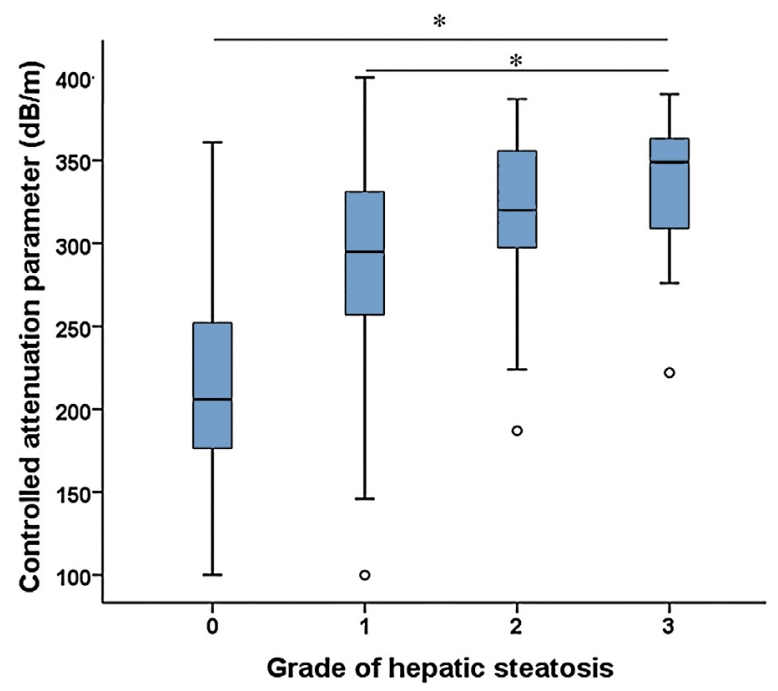

Figure 1 Controlled attenuation parameter values in different grades of hepatic steatosis in all patients $(n=462)$. *statistically significant $(P$ value $<0.05)$

invasive technique, can accurately assess hepatic steatosis in different etiologies with good accuracy. BMI and grade of hepatic steatosis (on liver biopsy) independently influence CAP values.

Hepatic steatosis is present in approximately $30 \%$ of the general population, and this percentage increases in patients with obesity and diabetes mellitus. ${ }^{6}$ The presence of steatosis influences the outcome of liver diseases, including NAFLD, ALD, HCV and HBV. ${ }^{3-5}$ Liver biopsy is the gold standard for hepatic steatosis estimation, but has its own limitations. Therefore, there is a need for noninvasive tests, which are safe, cheap, reliable, validated, can be done bedside if required, and performed repeatedly to assess the response after interventions. Prior studies have evaluated the role of CAP in patients with various etiologies of CLD. ${ }^{13,14,17,18}$ Our data suggests a high negative predictive value for CAP, which means that it can be used with good accuracy to rule out hepatic steatosis. The

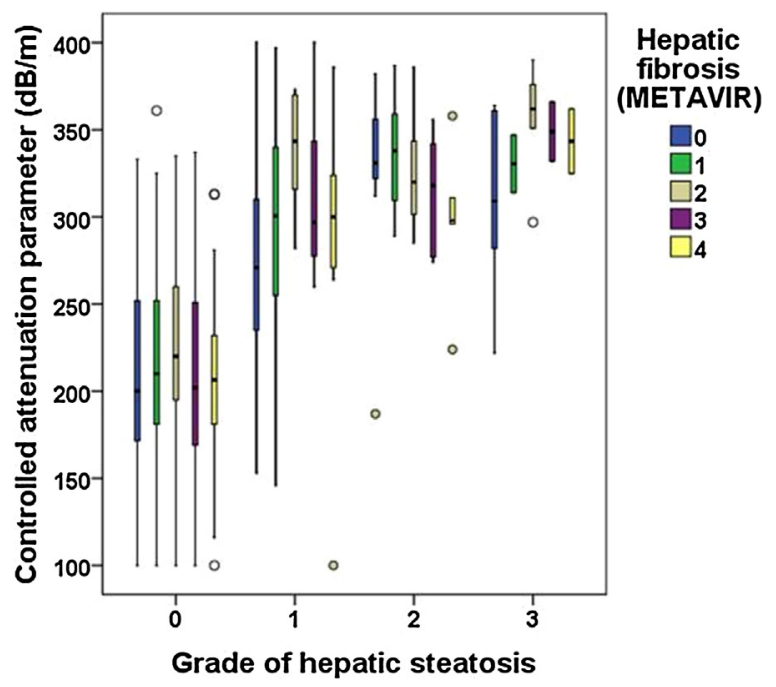

Figure 3 Controlled attenuation parameter value in different grades of hepatic steatosis across the various stages of hepatic fibrosis.

optimal cut-off values for detection of various grades of steatosis in our study were $\geq \mathrm{S} 1: 263.5 \mathrm{~dB} / \mathrm{m}, \geq \mathrm{S} 2$ : $287.5 \mathrm{~dB} / \mathrm{m}$ and S3: $296.5 \mathrm{~dB} / \mathrm{m}$. These cut-off values are similar to those reported by Myers et al. ${ }^{18}$ In our study, cut-off for steatosis grade $\geq \mathrm{S} 1$ was higher, whereas cut-off for $\geq \mathrm{S} 2$, and $\geq \mathrm{S} 3$ was similar to those reported by Kumar et al. ${ }^{19}$ The CAP cut-off values for differentiating various grades of hepatic steatosis were different between the various etiologies of liver disease possibly due to significant differences in the BMI. The median BMI of the NAFLD included in our cohort was higher as compared to those of Kumar et al. ${ }^{19} \mathrm{BMI}$ is an independent predictor of CAP values along with hepatic steatosis, which explains the higher CAP values in our cohort. ${ }^{19,20}$ In contrast, the CAP cut-offs in other studies were different. ${ }^{21,22}$ These differences in the cut-offs values can possibly be explained by differences in BMI, use of $\mathrm{M}$ or XL probe and differences in subcutaneous fat. ${ }^{23-25}$
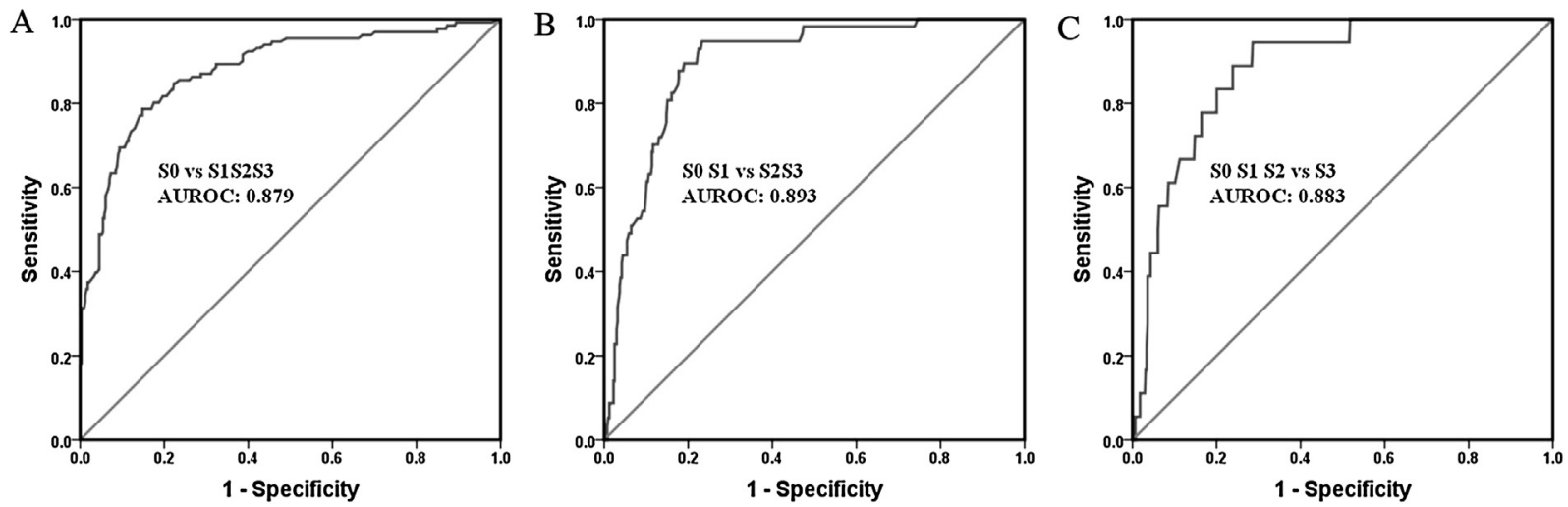

Figure 2 Receiver operating characteristic curve of controlled attenuation parameter and different grades of hepatic steatosis. S0: steatosis in 0-5\% of hepatocytes, S1: 6-33\%, S2: 34-66\% and S3: 67-100\%, AUROC: area under receiver operating characteristic curve. 
Table 3 Diagnostic Accuracy of Controlled Attenuation Parameter for Various Grades of Hepatic Steatosis Across Grades of Hepatic Fibrosis.

\begin{tabular}{|c|c|c|c|c|c|c|}
\hline & Overall & Fo & $\mathbf{F 1}$ & F2 & F3 & $\mathbf{F 4}$ \\
\hline $\begin{array}{l}\text { SO vs S1S2S3 } \\
\text { AUROC }\end{array}$ & $\begin{array}{l}0.879 \\
(0.842-0.916)\end{array}$ & $\begin{array}{l}0.836 \\
(0.764-0.907)\end{array}$ & $\begin{array}{l}0.867 \\
(0.790-0.944)\end{array}$ & $\begin{array}{l}0.965 \\
(0.925-1.00)\end{array}$ & $\begin{array}{l}0.934 \\
(0.870-0.998)\end{array}$ & $\begin{array}{l}0.898 \\
(0.79-1.000\end{array}$ \\
\hline Optimal cut-off & 263.5 & 243.0 & 262.0 & 277.5 & 270.5 & 263.0 \\
\hline Sensitivity & 81.7 (73.9-87.9) & $75.6(59.7-87.6)$ & $78.4(61.8-90.2)$ & $100.0(81.5-100.0)$ & $93.8(69.8-99.8)$ & 89.5 (66.9-98.7) \\
\hline Specificity & $80.4(75.7-84.5)$ & $73.5(65.3-80.7)$ & $77.9(67.0-86.6)$ & $87.8(73.8-95.9)$ & 85.7 (69.7-95.2) & 90.5 (77.4-97.3) \\
\hline PPV & $62.2(56.5-67.5)$ & $47.3(38.2-54.5)$ & $63.0(52.0-72.8)$ & $78.3(61.3-89.1)$ & $75.0(56.9-87.2)$ & $80.9(62.3-91.6)$ \\
\hline NPV & $91.7(88.5-94.1)$ & $90.9(85.3-94.5)$ & $88.2(80.1-93.3)$ & 100.0 & 96.8 (81.7-99.5) & $95.0(83.6-98.6)$ \\
\hline PLR & $\begin{array}{l}4.16 \\
(3.3-5.25)\end{array}$ & $\begin{array}{l}2.86 \\
(2.05-3.97)\end{array}$ & $\begin{array}{l}3.55 \\
(2.26-5.58)\end{array}$ & $\begin{array}{l}8.20 \\
(3.61-18.64)\end{array}$ & $\begin{array}{l}31.37 \\
(19.11-45.89)\end{array}$ & $\begin{array}{l}9.39 \\
(3.65-24.17)\end{array}$ \\
\hline NLR & $0.23(0.16-0.33)$ & $0.33(0.19-0.57)$ & $0.28(0.15-0.52)$ & - & $0.07(0.01-0.49)$ & $0.12(0.03-0.43)$ \\
\hline $\begin{array}{l}\text { SOS1 vs S2S3 } \\
\text { AUROC }\end{array}$ & $\begin{array}{l}0.893 \\
(0.855-0.931)\end{array}$ & $\begin{array}{l}0.885 \\
(0.791-0.979)\end{array}$ & $\begin{array}{l}0.900 \\
(0.842-0.958)\end{array}$ & $\begin{array}{l}0.906 \\
(0.832-0.980)\end{array}$ & $\begin{array}{l}0.885 \\
(0.790-0.981)\end{array}$ & $\begin{array}{l}0.854 \\
(0.737-0.971)\end{array}$ \\
\hline Optimal cut-off & 287.5 & 275.5 & 302.5 & 296.0 & 272.5 & 288.5 \\
\hline Sensitivity & 87.7 (76.3-94.9) & $87.5(61.7-98.5)$ & 84.6 (54.6-98.1) & 83.3 (51.6-97.9) & $100.0(63.1-100.0)$ & $87.5(47.4-99.7)$ \\
\hline Specificity & $82.2(78.1-85.8)$ & $82.0(75.2-87.6)$ & $85.2(76.7-91.4)$ & $83.0(69.2-92.4)$ & $74.4(58.8-86.5)$ & $84.9(72.4-93.3)$ \\
\hline PPV & $40.9(35.5-46.7)$ & $32.6(24.9-41.3)$ & $42.3(30.3-55.3)$ & $55.6(38.8-71.2)$ & $42.1(30.4-54.8)$ & $46.7(30.5-63.6)$ \\
\hline NPV & 97.9 (95.9-98.9) & $98.5(94.7-99.6)$ & 97.7 (92.3-99.4) & $95.1(84.5-98.6)$ & 100.0 & $97.8(87.8-99.7)$ \\
\hline PLR & $4.93(3.92-6.22)$ & $4.86(3.33-7.09)$ & $5.71(3.38-9.60)$ & $4.90(2.48-9.66)$ & $3.91(2.35-6.51)$ & $5.80(2.91-11.56)$ \\
\hline NLR & $0.15(0.07-0.30)$ & $0.15(0.04-0.56)$ & $0.18(0.05-0.65)$ & $0.20(0.06-0.72)$ & - & $0.15(0.02-0.92)$ \\
\hline $\begin{array}{l}\text { SOS1S2 vs S3 } \\
\text { AUROC }\end{array}$ & $\begin{array}{l}0.883 \\
(0.822-0.944)\end{array}$ & $\begin{array}{l}0.852 \\
(0.735-0.969)\end{array}$ & $\begin{array}{l}0.855 \\
(0.773-0.937)\end{array}$ & $\begin{array}{l}0.915 \\
(0.823-1.000)\end{array}$ & $\begin{array}{l}0.918 \\
(0.810-1.000)\end{array}$ & $\begin{array}{l}0.949 \\
(0.891-1.000)\end{array}$ \\
\hline Optimal cut-off & 296.5 & 275.5 & 311.0 & 296.0 & 331.5 & 320.0 \\
\hline Sensitivity & $\begin{array}{l}83.3 \\
(58.6-96.4)\end{array}$ & $\begin{array}{l}85.7 \\
(42.1-99.6)\end{array}$ & $\begin{array}{l}100.0 \\
(15.8-100.0)\end{array}$ & $\begin{array}{l}100.0 \\
(47.8-100.0)\end{array}$ & $\begin{array}{l}100.0 \\
(15.8-100.0)\end{array}$ & $\begin{array}{l}100.0 \\
(15.8-100.0)\end{array}$ \\
\hline Specificity & $80.0(75.9-83.6)$ & $78.2(71.3-84.2)$ & $81.2(72.8-88.0)$ & $75.9(62.4-86.5)$ & $85.7(72.8-94.1)$ & $93.2(83.5-98.1)$ \\
\hline PPV & $14.4(11.3-18.2)$ & $13.9(9.7-19.7)$ & $8.7(6.1-12.3)$ & $27.8(19.3-38.2)$ & $22.2(12.6-36.2)$ & $33.3(16.3-56.3)$ \\
\hline NPV & $99.2(97.7-99.7)$ & 99.3 (95.6-99.9) & 100.0 & 100.0 & 100.0 & 100.0 \\
\hline PLR & $4.16(3.15-5.49)$ & $3.94(2.60-5.97)$ & $5.33(3.63-7.84)$ & $4.15(2.59-6.67)$ & $7.00(3.53-13.90)$ & $14.75(5.73-37.99)$ \\
\hline NLR & $0.21(0.07-0.59)$ & $0.18(0.03-1.12)$ & - & - & - & - \\
\hline
\end{tabular}

Abbreviations: AUROC, area under receiver operator characteristic curve; PPV, positive predictive value; NPV, negative predictive value; LR, likelihood ratio; S, steatosis.

Classically, hepatic steatosis is graded on histology by pathologists by visual estimation of extent of hepatic steatosis. CAP gives an objective number which can be used to quantify hepatic steatosis and in turn, can be used objectively for following up patients with hepatic steatosis. An ideal test is one which is not be affected by other factors. Our data also suggests that CAP is a good test for differentiating patients with hepatic steatosis of $\geq \mathrm{S} 1, \geq \mathrm{S} 2$, and $\geq \mathrm{S} 3$, irrespective of underlying fibrosis in the liver. There was no significant difference in CAP values in a different stages of fibrosis, when compared in patients with similar grade of steatosis. Practically, CAP can be used for assessing hepatic steatosis in all etiologies and stages of fibrosis. The ease of use of this technique at bed-side, availability of immediate results and the overall low cost as compared to other non-invasive techniques make this an important tool in the assessment of hepatic steatosis.
Our data also suggests that CAP performs best in differentiating patients with no steatosis and presence of any steatosis. Furthermore, CAP performs better in differentiating patients with a difference of 2 or more grades of hepatic steatosis (i.e. differentiating between S0/S2 grades and S0/S3 grades) as compared to differentiating 1 grade of hepatic steatosis (i.e. differentiating between S0 vs S1). Our results are similar to those reported in a prior study in HCV patients and another Indian study involving patients with varied etiologies of liver disease. ${ }^{14,19}$

However, CAP performed poorly in differentiating patients with S1 and S2 hepatic steatosis; S2 and S3 hepatic steatosis. Although the number of patients with S3 hepatic steatosis was less in our cohort, in previous published studies also similar observations were noted, ${ }^{14,18,19,21,22,26,27}$ suggesting the fact that CAP is 
Table 4 Diagnostic Accuracy of Controlled Attenuation Parameter for Differentiating Various Grades of Steatosis.

\begin{tabular}{lllllll}
\hline & \multicolumn{1}{c}{ S0 vs S1 } & \multicolumn{1}{c}{ S0 vs S2 } & \multicolumn{1}{c}{ S0 vs S3 } & \multicolumn{1}{c}{ S1 vs S2 } & \multicolumn{1}{c}{ S1 vs S3 } & \multicolumn{1}{c}{ S2 vs S3 } \\
\hline AUROC & 0.831 & 0.936 & 0.954 & 0.659 & 0.715 & 0.600 \\
& $(0.776-0.886)$ & $(0.895-0.976)$ & $(0.910-0.997)$ & $(0.558-0.759)$ & $(0.594-0.837)$ & $(0.434-0.766)$ \\
Optimal cut-off & 252.5 & 284.5 & 287.5 & 310.5 & 324.5 & 331.5 \\
Sensitivity & $78.4(67.3-87.1)$ & $89.7(75.8-97.1)$ & $88.9(65.3-98.6)$ & $66.7(49.8-80.9)$ & $66.7(40.9-86.7)$ & $61.1(35.8-82.7)$ \\
Specificity & $76.4(71.5-80.9)$ & $89.1(85.3-92.3)$ & $90.9(86.9-93.6)$ & $62.2(50.1-73.2)$ & $71.6(59.9-81.5)$ & $59.0(42.1-74.4)$ \\
PPV & $42.7(37.2-48.3)$ & $49.3(41.2-57.4)$ & $34.0(26.2-42.8)$ & $48.2(39.2-57.3)$ & $36.7(25.9-48.2)$ & $40.7(28.9-53.8)$ \\
NPV & $0.28(0.18-0.44)$ & $98.7(96.7-99.5)$ & $99.3(97.6-99.8)$ & $77.9(68.7-85.1)$ & $89.8(81.9-94.5)$ & $76.7(63.5-86.1)$ \\
LR+ & $3.33(2.65-4.18)$ & $8.25(5.96-11.43)$ & $9.49(6.54-13.78)$ & $1.76(1.22-2.54)$ & $2.35(1.44-3.83)$ & $1.49(0.88-2.52)$ \\
LR- & $0.28(0.18-0.44)$ & $0.12(0.05-0.29)$ & $0.12(0.03-0.45)$ & $0.54(0.33-0.86)$ & $0.47(0.24-0.91)$ & $0.66(0.35-1.25)$ \\
\hline
\end{tabular}

Abbreviations: AUROC, area under receiver operator characteristic curve; PPV, positive predictive value; NPV, negative predictive value; LR, likelihood ratio; S, steatosis.

suboptimal in differentiating between moderate and severe grades of hepatic steatosis. These results gain importance while assessing potential liver donors. Majority of the liver transplants in India are living donor related. Liver graft steatosis is associated with increased complications, ${ }^{28}$ and presence of significant fat is considered a contraindication for living donation. Therefore, it is essential to quantify fat accurately. Our data suggests that CAP may not be accurate in differentiating S2 and S3 steatosis, hence liver biopsy may still be of use in this subgroup of patients. MRI based proton density fat fraction (MRIPDFF) has a very good correlation with quantification of steatosis by liver histology. MRI-PDFF is a noninvasive and accurate method for differentiation of different grades of hepatic steatosis. ${ }^{29}$ MRI-PDFF is presently being used in clinical trials and donor evaluation algorithms. Thus, suboptimal utility of the CAP in differentiating between consequent grades of steatosis can be circumvented by MRI-PDFF.

On multivariate analysis, we found only BMI and hepatic steatosis to be independent predictors of CAP. The presence of metabolic syndrome was not significantly associated with CAP in the overall cohort. Metabolic syndrome is more common in patients with NAFLD as compared to viral hepatitis. ${ }^{30}$ Our cohort had more patients with viral hepatitis as compared to NAFLD.

Our study has limitations. We could not compare CAP to other non-invasive tools like NAFLD fibrosis score, fatty liver index, enhanced liver function score, and MRI-PDFF for diagnostic accuracy of steatosis and fibrosis, as these tests are not routinely available in our hospital. We also did not take into account the skin-liver capsule distance which could have influenced the results of fibroscan. We included a heterogeneous group of etiologies in our cohort of chronic liver disease, which may have influenced the CAP values in this group.

In conclusion, CAP can quantitatively estimate hepatic steatosis with good accuracy across various etiologies and irrespective of extent of hepatic fibrosis.

\section{AUTHORS' CONTRIBUTIONS}

Gyanranjan Rout: acquisition of data; analysis and interpretation of data; drafting of manuscript; critical revision of manuscript.

Saurabh Kedia: acquisition of data; drafting of manuscript.

Baibaswata Nayak: acquisition of data; analysis of blood samples; drafting of manuscript.

Rajni Yadav: analysis of liver biopsy; drafting of manuscript.

Prasenjit Das: analysis of liver biopsy; drafting of manuscript.

Subrat Kumar Acharya: interpretation of data; drafting of manuscript.

Deepak Gunjan: interpretation of data; drafting of manuscript.

Vishwajeet Singh: statistical analysis; interpretation of the data.

Mousumi Mahanta: acquisition of data; drafting of manuscript.

Swatantra Gupta: acquisition of data; drafting of manuscript.

Sandeep Aggarwal: acquisition of data; drafting of manuscript.

Shalimar: study concept and design; interpretation of data; critical revision of manuscript for important intellectual content.

\section{CONFLICTS OF INTEREST}

The authors have none to declare.

\section{ACKNOWLEDGEMENT}

Mr. Anurag and Ms. Manisha Kumari for data maintenance.

\section{REFERENCES}

1. Angulo P. Nonalcoholic fatty liver disease. $N$ Engl J Med. 2002;346:1221-1231. 
2. Kleiner DE, Brunt EM, Van Natta M, et al. Design and validation of a histological scoring system for nonalcoholic fatty liver disease. Hepatology. 2005;41:1313-1321.

3. Jin X, Chen Y, Yang Y, Li Y, Zheng L, Xu C. Association between hepatic steatosis and entecavir treatment failure in Chinese patients with chronic hepatitis B. PLoS ONE. 2012;7:e34198.

4. Leandro G, Mangia A, Hui J, et al. Relationship between steatosis, inflammation, and fibrosis in chronic hepatitis C: a meta-analysis of individual patient data. Gastroenterology. 2006;130:16361642.

5. Kurosaki M, Hosokawa T, Matsunaga K, et al. Hepatic steatosis in chronic hepatitis $\mathrm{C}$ is a significant risk factor for developing hepatocellular carcinoma independent of age, sex, obesity, fibrosis stage and response to interferon therapy. Hepatol Res. 2010;40:870-877.

6. Duseja A, Singh SP, Saraswat VA, et al. Non-alcoholic fatty liver disease and metabolic syndrome-position paper of the Indian National Association for the Study of the Liver, Endocrine Society of India, Indian College of Cardiology and Indian Society of Gastroenterology. J Clin Exp Hepatol. 2015;5:51-68.

7. Rockey DC, Caldwell SH, Goodman ZD, Nelson RC, Smith AD, American Association for the Study of Liver Diseases. Liver biopsy. Hepatology. 2009;49:1017-1044.

8. Lee SS, Park SH, Kim HJ, et al. Non-invasive assessment of hepatic steatosis: prospective comparison of the accuracy of imaging examinations. J Hepatol. 2010;52:579-585.

9. Cesbron-Métivier E, Roullier V, Boursier J, et al. Noninvasive liver steatosis quantification using MRI techniques combined with blood markers. Eur J Gastroenterol Hepatol. 2010;22:973-982.

10. Longo R, Pollesello P, Ricci C, et al. Proton MR spectroscopy in quantitative in vivo determination of fat content in human liver steatosis. J Magn Reson Imaging JMRI. 1995;5:281-285.

11. Thomsen C, Becker U, Winkler K, Christoffersen P, Jensen M, Henriksen O. Quantification of liver fat using magnetic resonance spectroscopy. Magn Reson Imaging. 1994;12:487-495.

12. Singh S, Muir AJ, Dieterich DT, Falck-Ytter YT. American Gastroenterological Association Institute technical review on the role of elastography in chronic liver diseases. Gastroenterology. 2017;152:1544-1577.

13. Friedrich-Rust M, Romen D, Vermehren J, et al. Acoustic radiation force impulse-imaging and transient elastography for non-invasive assessment of liver fibrosis and steatosis in NAFLD. Eur J Radiol. 2012;81(3):e325-e331.

14. Sasso M, Tengher-Barna I, Ziol M, et al. Novel controlled attenuation parameter for noninvasive assessment of steatosis using Fibroscan(®): validation in chronic hepatitis C. J Viral Hepat. 2012;19(4):244-253.

15. Karlas T, Petroff D, Sasso M, et al. Individual patient data metaanalysis of controlled attenuation parameter (CAP) technology for assessing steatosis. J Hepatol. 2017;66(5):1022-1030.

16. Bedossa P, Poynard T. An algorithm for the grading of activity in chronic hepatitis C. The METAVIR Cooperative Study Group. Hepatology. 1996;24:289-293.
17. Garg H, Aggarwal S, Shalimar. et al. Utility of transient elastography (fibroscan) and impact of bariatric surgery on nonalcoholic fatty liver disease (NAFLD) in morbidly obese patients. Surg Obes Relat Dis. 2018;14:81-91.

18. Myers RP, Pollett A, Kirsch R, et al. Controlled Attenuation Parameter (CAP): a noninvasive method for the detection of hepatic steatosis based on transient elastography. Liver Int. 2012;32: 902-910.

19. Kumar M, Rastogi A, Singh $T$, et al. Controlled attenuation parameter for non-invasive assessment of hepatic steatosis: does etiology affect performance? J Gastroenterol Hepatol. 2013;28: 1194-1201.

20. Jung KS, Kim BK, Kim SU, et al. Factors affecting the accuracy of controlled attenuation parameter (CAP) in assessing hepatic steatosis in patients with chronic liver disease. PLOS ONE. 2014;9: doi:10.1371/journal.pone.0098689.

21. Chon YE, Jung KS, Kim SU, et al. Controlled attenuation parameter (CAP) for detection of hepatic steatosis in patients with chronic liver diseases: a prospective study of a native Korean population. Liver Int. 2014;34:102-109.

22. de Lédinghen V, Vergniol J, Foucher J, Merrouche W, le Bail B. Noninvasive diagnosis of liver steatosis using controlled attenuation parameter (CAP) and transient elastography. Liver Int. 2012;32:911-918.

23. Shen F, Zheng R, Shi J, et al. Impact of skin capsular distance on the performance of controlled attenuation parameter in patients with chronic liver disease. Liver Int. 2015;35:2392-2400.

24. Myers RP, Pomier-Layrargues G, Kirsch R, et al. Feasibility and diagnostic performance of the FibroScan XL probe for liver stiffness measurement in overweight and obese patients. Hepatology. 2012;55:199-208.

25. Wong VW., Vergniol J, Wong GL., et al. Liver stiffness measurement using $\mathrm{XL}$ probe in patients with nonalcoholic fatty liver disease. Am J Gastroenterol. 2012;107:1862-1871.

26. Masaki K, Takaki S, Hyogo H, et al. Utility of controlled attenuation parameter measurement for assessing liver steatosis in Japanese patients with chronic liver diseases. Hepatol Res. 2013;43:1182-1189.

27. Shen F, Zheng R-D, Mi Y-Q, et al. Controlled attenuation parameter for non-invasive assessment of hepatic steatosis in Chinese patients. World J Gastroenterol. 2014;20:4702-4711.

28. McCormack L, Dutkowski P, El-Badry AM, Clavien P-A. Liver transplantation using fatty livers: always feasible? J Hepatol. 2011;54:1055-1062.

29. Park CC, Nguyen P, Hernandez C, et al. Magnetic resonance elastography vs transient elastography in detection of fibrosis and noninvasive measurement of steatosis in patients with biopsy-proven nonalcoholic fatty liver disease. Gastroenterology. 2017;152:598-607.e2.

30. Tsochatzis E, Papatheodoridis GV, Manesis EK, Kafiri G, Tiniakos DG, Archimandritis AJ. Metabolic syndrome is associated with severe fibrosis in chronic viral hepatitis and non-alcoholic steatohepatitis. Aliment Pharmacol Ther. 2008;27:80-89. 